\title{
Effect of acetone feeding on alcohol dehydrogenase activity in the olive fruit fly, Bactrocera oleae
}

\author{
N Cosmidis ${ }^{1}, \mathrm{M}$ Loukas $^{1}$, V Peppa ${ }^{1}, \mathrm{G}$ Goulielmos $^{1}$ and E Zouros ${ }^{2}$ \\ ${ }^{1}$ Department of Agriculture Biotechnology, Agricultural University of Athens, Iera Odos 75, Athens, Greece; ${ }^{2}$ Department of \\ Biology, University of Crete, Iraklion, Crete, Greece
}

The purpose of this study is to demonstrate a clear connection between the presence of acetone in larval diet and alcohol dehydrogenase (ADH) activity in laboratory raised populations of Bactrocera oleae. ADH activity of $B$. oleae is depressed in acetone-impregnated diets. At the same time the change of activity is accompanied by a change in the relative proportions of the multiple forms of $\mathrm{ADH}$. The bulk of activity in the most cathodally migrating form is lost, and all the activity becomes localized in the less cathodally migrating forms of the enzyme. Moreover, ADH activity, expressed in vivo, appears to drop after exposure to ace- tone, as shown by the fact that larvae become less sensitive to pentenol poisoning. Our results show clear selective differences imposed by acetone on three homozygous genotypes involving the ADH alleles $F, S$ and I in B. oleae. The directions of these differences were found to vary with the fitness component under test. Acetone treatment seems to affect developmental time and larva's viability as well as allele frequencies of $\mathrm{ADH}$ under artificial rearing. The effect of acetone on the maintenance of $\mathrm{ADH}$ polymorphism in artificially reared populations of $B$. oleae is further discussed. Heredity (2002) 89, 453-459. doi:10.1038/sj.hdy.6800163

Keywords: alcohol dehydrogenase; Bactrocera oleae; acetone; multiple forms

\section{Introduction}

Throughout the Mediterrannean basin, where almost all of the world's olive oil is produced, Bactrocera oleae is considered the most serious insect pest of olives. In several overlapping generations it attacks olives from early summer up to harvest time. In nature, the olive fruit fly is practically monophagus, ovipositing, feeding and growing in the olive mesocarp of the fruit of cultivated and wild olives. At present, almost 140 million olive trees are cultivated in Greece. Crop losses due to B. oleae infestation can reach $30 \%$ of the production in untreated areas. The main component of damage caused by $B$. oleae is the preharvest fruit drop of the infested fruits. The olive fruit fly is being artificially cultured on a small scale in the Department of Genetics at the Agricultural University of Athens.

The objective of the undertaking is to gain insight into the genetic structure of the insect because it will greatly facilitate a more efficient and environmentally safe fight against this pest. It is highly recommended to replace the chemical control of the fly, now in general use, with an ecologically less damaging programme which will rely heavily on autocidal control applying the Sterile Insect Technique (Economopoulos et al, 1977).

The introduction of $B$. oleae flies from the wild into the laboratory, for use in developing and applying SIT, results in changes concerning the genetic structure,

Correspondence: N Cosmidis, Department of Agriculture Biotechnology, Agricultural University of Athens, Iera Odos 75, Athens, Greece. Email: bgen7con@aua.gr

Received 10 July 2001; accepted 9 July 2002 physiology, behaviour and reproductive biology of the insect. The most profound change among the many different loci affected deals with the alcohol dehydrogenase $(\mathrm{ADH})$ polymorphism. The end product of this process is an insect that differs from that in the wild in many important aspects. The dramatic response of the ADH polymorphism to artificial rearing always appears simultaneously with major changes in the life-history of the insect. This can hardly be mere coincidence. Rather, it suggests a correlated response. In fact, attempts to restore the ADH alleles' equilibrium towards the one found in nature might improve greatly the quality of the laboratory colonies. This point must be kept in mind because critical ecological, behavioural and genetic characteristics must be preserved during laboratory culture to assure that flies do not diverge significantly from those in nature.

The principal function of the enzyme ADH (EC 1.1.1.1.) in insects' metabolism is the catalysis of the reversible conversion of various alcohols generated by microbial fermentation in larval and adult feeding sites to their corresponding aldehydes and ketones. All natural populations of the olive fruit fly $B$. oleae examined so far are polymorphic for the locus ADH. Allele F (Fast) occurs in frequencies of about 0.4 and allele $S$ (Slow) in 0.6. A third allele I (Intermediate) occurs in frequencies less than 0.01. In laboratory colonies maintained on artificial medium the frequency of the rare allele I increases to about 0.3 and that of allele $S$ decreases to 0.3 . Allele F's frequency is unaffected. This change becomes established after three to four generations and is indifferent to the geographic origin of the natural populations or the temperature under which the population is kept in the laboratory 
(Zouros et al, 1982; Economopoulos and Loukas, 1986). Insects raised on ripe olive fruit under laboratory conditions do not show any differences in the allele frequencies when compared with natural populations. The changes observed are solely the result of the artificial diet on which the larvae are maintained. Results of an extensive study designed to estimate fitness components of the various genotypes at the $\mathrm{ADH}$ locus and to identify the stage in the life cycle of the insect at which selection pressure is most intense have revealed significant differences among genotypes in egg-to-adult viability and in egg-to-adult emergence time (Cosmides et al, 1997).

The ADH enzyme from $B$. oleae has a dimeric structure with a subunit mass of $26 \mathrm{kDa}$ (Mazi et al, 1998a). In each homozygous strain of B. oleae three molecular forms of the enzyme are present. Schwartz et al (1975) and Schwartz and Sofer (1976) have confirmed that, in Drosophila melanogaster, these additional forms of the enzyme originate from the tight, but non-covalent, binding of one or two molecules of a NAD-carbonyl addition compound (Everse et al, 1971) to the native enzyme. Schwartz and Sofer (1976) found that exposing D. melanogaster flies to a variety of carbonyl compounds, whose structures were similar to substrates of ADH, resulted in an increase in activity of those forms postulated to carry a NAD-carbonyl compound and a decrease in activity in the major form of the enzyme, thought to be free of the compound. They termed this process 'conversion'. Moreover, they demonstrated that conversion resulted in a lowering of total ADH activity in homogenates of whole flies without a change in the total ADH protein. Earlier studies in Drosophila have demonstrated that when adult flies are exposed to $0.5 \%$ acetone their ADH activity (measured spectrophotometrically) is severely depressed (Papel et al, 1979). They have also confirmed that ADH function is impaired by acetone in vivo as well, as shown by insensitivity to pentenol treatment in those flies with low levels of ADH activity. Pentenol (1-pentene-3-ol) is a secondary unsaturated alcohol and was chosen for study because Sofer and Hatkoff (1972) suggested that, in vivo, ADH oxidizes it into a lethal ketone, ethyl vinyl ketone (pentenone), which is a compound two or three times more toxic than the former (Garrido et al, 1988). This procedure preferentially kills flies with high levels of ADH activity, because pentenone does not appear to be metobolized by Drosophila and its accumulation is highly toxic to flies. A number of questions related to this model arise concerning $B$. oleae. Five that we address in this paper, based on previous work in Drosophila, are as follows: (1) can we interpret the diet-induced changes in the distribution of multiple forms of $\mathrm{ADH}$ in $B$. oleae according to the respective model in Drosophila? (2) Is acetone capable of increasing the efficacy of the conversion process and thereby effectively eliminating $\mathrm{ADH}$ activity? (3) Can the change of $\mathrm{ADH}$ activity be shown to occur in vivo as well as in vitro? (4) Does this change of $\mathrm{ADH}$ activity modify the physiology of the laboratory flies with respect to their developmental times and egg-to-adult survival? (5) Are $\mathrm{ADH}$ allele frequencies of laboratory populations reared for several generations on standard larval diet affected when acetone is added in the larval diet?

\section{Materials and methods}

\section{Artificial rearing of $B$. oleae}

All experimental populations were maintained according to the protocol of Tsitsipis (1983) for the rearing of the olive fruit fly under artificial conditions.

\section{Scoring of ADH allozyme polymorphism}

A detailed description of the ADH polymorphism (which is controlled by an autosomal locus) and the method of its detection is given in Zouros et al (1982).

\section{Production of homozygous strains}

The olive fruit fly stocks used in this study were described in detail by Cosmides et al (1997). They originated from two three-allele laboratory 'colonies' that were established from wild B. oleae collected from two different locations in Attica, Greece. From each colony, three 'lines', each homozygous for a different allele (S, I and $\mathrm{F}$ ) at the ADH locus, were extracted through singlepair brother/sister matings. For this study we produced three homozygous 'experimental stocks' (SS, II, FF) by putting in the same chamber 1000 virgin females and 1000 virgin males from each of the two lines of same genotype.

Experimenting with insects of the same genotype extracted from two different populations reduces the danger of inbreeding that may accumulate during the extraction and maintenance of the pure lines, and also of linkage disequilibrium of $\mathrm{ADH}$ alleles with other genes affecting fitness components.

\section{Enzyme activity and gel electrophoresis}

Details of the assay for measuring $\mathrm{ADH}$ activity are described in Mazi et al (1998b). The protein concentration was determined by the method of Bradford (1976) using bovine serum albumin (fraction V) as standard.

$\mathrm{ADH}$ allozymes were resolved by starch gel electrophoresis using Poulik's (1957) discontinuous buffer system.

\section{Effect of acetone on two fitness components}

Genotype specific viability: Insects of the three different $\mathrm{ADH}$ homozygous genotypes were left to oviposit in specially designed layers of paraffin. Eggs derived from each genotype were divided into three groups. The first group was placed on a standard larval artificial diet (control), the second on a diet to which $0.5 \%$ acetone was added and the third on a diet with $1 \%$ acetone. Egg density was five eggs per gram of diet. Each diet (treatment) was put in round plastic containers of $7 \mathrm{~cm}$ lower diameter, $9 \mathrm{~cm}$ upper diameter and $4 \mathrm{~cm}$ height, covered with plastic lids. Eight replicates were made in each case. Adult recovery was estimated based on the number of eggs compared to the number of adults produced. Each replicate started with 200 eggs.

Developmental time: Differences in developmental time among the three homozygous genotypes grown on an artificial diet with or without acetone were examined in five replicates for each treatment. Egg density was five eggs per gram of diet. Each replicate started with 200 eggs. Eggs from each genotype were placed in all types 
of diet (standard, acetone $0.5 \%$ and $1 \%$ ) on the same day and adults eclosing on each consecutive day were counted.

\section{ADH activity}

The effect of acetone on ADH activity was evaluated at three different stages: larvae, pupae and adults. To establish the relation between acetone and in vitro $\mathrm{ADH}$ activity, samples of all ADH homozygous genotypes were taken each day, starting from the 7th day after egg eclosion (late second instar larvae) until the 20th day (2 to 4-hour old adults).

Samples were collected from regular food, food containing $0.5 \%$ acetone and food containing $1 \%$ acetone. For each day larvae were removed from a particular plastic cup (replicate) which was not used for this purpose any more. This scheme was adopted to avoid larvae density disturbance which, in turn, could possibly affect ADH activity. Five extracts were tested for each genotype, day and food, and each one was assayed twice with isopropanol as substrate.

\section{Pentenol sensitivity of acetone-treated larvae}

This experiment was designed in order to provide further evidence that acetone treatment alters ADH activity not only in vitro but in vivo as well. Larvae from each genotype were independently raised on normal food and on food supplemented with $0.5 \%$ acetone. Eight-day-old larvae of each treatment were transferred in hermetically closed vials and exposed to a $4 \%$ pentenol solution. After $40 \mathrm{~min}$ in this solution, the mortality and the ADH activity of survivors were determined. A set of eight replicates were tested for each condition, each replicate (vial) containing 20 larvae.

In order to provide further evidence that acetone reduces significantly ADH activity in vivo, we conducted another experiment. Bactrocera oleae larvae 5, 6 and 7 days old already reared on control, $0.5 \%$ acetone and $1 \%$ acetone diets were transferred on to a diet with $0.025 \%$ pentenol but without acetone. The percentage viability from larvae to adults was measured in these nine different agediet combinations. Each treatment was tested four times with a sample of 100 larvae for each replicate.

Meanwhile, larvae reared for 7 days on diets supplemented with $0.5 \%$ acetone were divided into two groups. The first group was placed on a diet with $0.5 \%$ acetone and $0.025 \%$ pentenol and the second group on a diet with $0.025 \%$ pentenol but without acetone. The same procedure was followed with diets supplemented with $1 \%$ acetone in the first and also in the second part of the experiment. If $B$. oleae $\mathrm{ADH}$ transforms pentenol in pentenone in vivo and if the latter compound does accumulate in the larva, a reduction of ADH activity levels caused by acetone must imply a decrease in the sensitivity to pentenol.

\section{Effect of acetone on ADH allele frequencies}

A B. oleae colony maintained in the laboratory for more than 50 generations was split into two lines. Larvae of the first line were fed on a diet including $0.5 \%$ acetone and those of the second line on a diet including $1 \%$ acetone. We followed $\mathrm{ADH}$ frequencies in each line for two subsequent generations.

\section{Results}

The effect of long-term exposure to acetone during the whole larval cycle for the ADH homozygous genotypes of B. oleae was studied with respect to ADH activity, larval viability and developmental time. For both acetone concentrations tested (0.5 and $1 \%)$ ADH activities during the larval stage in all genotypes decreased markedly (5594\%) but, during the pupal stage, the situation was found to be somewhat different. Differences in pupal ADH activity between individuals fed on a regular or on an acetone diet were diminished, compared with the differences in larval ADH activity and, in some cases, in ADH activity in pupae derived from the acetone diet outperformed the corresponding pupal ADH activity on a regular diet. We do not know the rate of ADH gene expression during development and to what extent this is connected to the ADH activity oscillations observed between consecutive days. A striking point in Figure 1 is that when larvae are ready to pupate (day 10), $\mathrm{ADH}$ activity of all genotypes and treatments decreased very strongly. These diet-induced changes in $\mathrm{ADH}$ activity seem to be related to electrophoretic alterations observed when larvae (with all known electrophoretic variants of ADH) are subjected to acetone treatment. As expected, the most cathodally migrating form disappears, while activity rises for the less cathodal migrating form (Figure 2). The least cathodal form is very faint when larvae are raised on regular food and does not appear in the picture.

However, these data were obtained by assessing enzyme activity in vitro after homogenization of the larvae. We wondered whether the in vitro assays reflected a real decrease in ADH activity in vivo. We studied this by subjecting acetone-treated larvae to pentenol treatment. Table 1 shows the ADH activities and mortalities of larvae of ADH homozygous genotypes after different pretreatment regimes. FF and SS larvae fed on a diet without acetone and exposed to $4 \%$ pentenol for $40 \mathrm{~min}$ manifested significantly higher mortalities compared to larvae raised on a diet with $0.5 \%$ acetone. The data in Table 1 suggest a genotypic effect within each one of the tested homozygous genotypes. The II larva tolerance to pentenol treatment is not significantly different when larvae fed on the regular and on the $0.5 \%$ acetone diet are compared, while SS larvae exhibited a three-fold higher mortality on pentenol than did FF larvae when both genotypes were fed on a diet with $0.5 \%$ acetone, though SS ADH activity was lower than that of FF ones,. The detrimental effect of pentenol on $B$. oleae larvae fed for a period of time on diets with or without acetone are shown in Table 2. In the case of the regular diet, it is clear that viability is positively correlated with the age of the larvae. In fact, older larvae will spend a shorter length of time on the diet with $0.025 \%$ pentenol and consequently will consume less pentenol before pupation occurs.

With regards to proportions of adults recovered the 7day-old larvae previously reared on $0.5 \%$ acetone diet not only were reduced by pentenol but also were lower than those of larvae of the same age reared on the regular diet. This is not surprising, as it is known that developmental times on $0.5 \%$ acetone are greater than those in the absence of acetone (Table 3). Therefore, larvae pretreated with $0.5 \%$ acetone up to the 7 th day of age were for a longer time susceptible to pentenol poisoning. As expected, due to the different developmental time, 7-day- 
FF

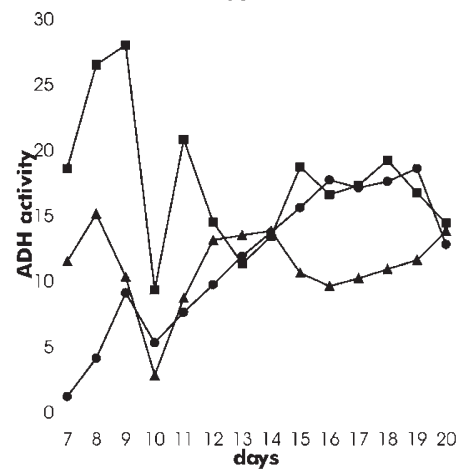

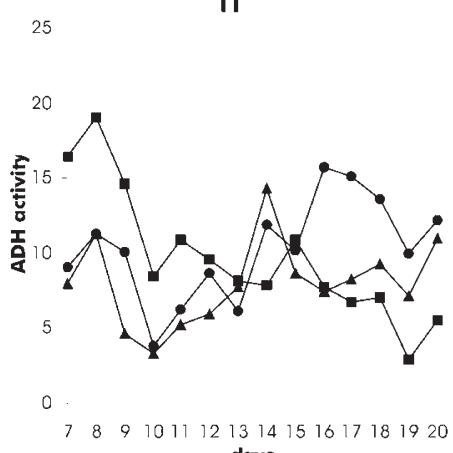

days

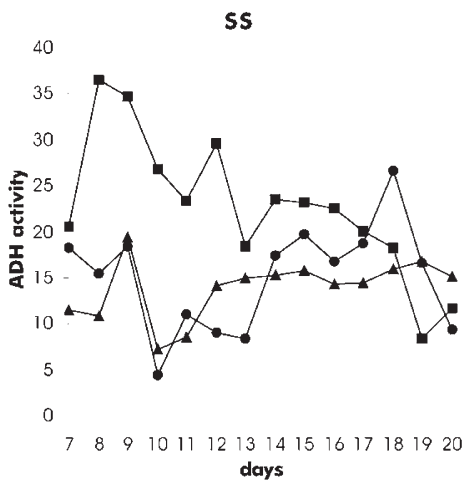

Figure 1 Mean in vitro ADH activities, measured in individuals of different genotypes (FF, II and SS) and submitted to different tratments ( $\mathbf{\square}$ regular diet, $\boldsymbol{\Delta} 0.5 \%$ acetone diet, $1 \%$ acetone diet). Activities are expressed as $\mu$ mol NADH $\times 10^{2} \mathrm{~min}^{-1} \mathrm{mgr}$ protein. Days $7-10$, 11-19 and 20 are larval, pupal and adult stage respectively. The number of replicates was 5.

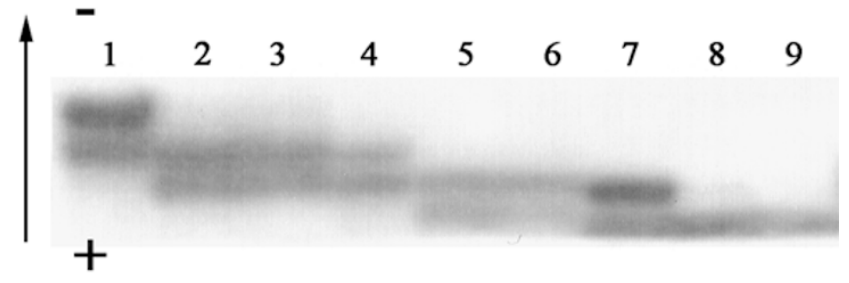

Figure 2 Electrophoretic patterns of ADH-F, ADH-I and ADH-S allozymes in Bactrocera oleae from extracts of larvae after 8 days of exposure to regular diet $(1,4$ and 7$)$, to $0.5 \%$ acetone diet $(2,5$ and 8 ) and to $1 \%$ acetone diet (3, 6 and 9). The following electrophoretic genotypes were used: ADH-FF (1, 2 and 3), ADH-II (4, 5 and 6) and ADH-SS (7, 8 and 9).

Table 1 Effect of acetone treatment on toxicity of pentenol. Larvae fed 8 days on regular diet or on diet containing $0.5 \%$ acetone were exposed to the vapor from a $4 \%$ solution of pentenol for $40 \mathrm{~min}$. Mortalities are expressed as the proportion of dead larvae. These proportions are the mean percentages of eight replicates (20 larvae per replicate)

\begin{tabular}{lccc}
\hline Genotype & Treatment & ADH activity a & Mortality $\%$ \\
\hline FF & regular diet & 18.0 & $13 \mathrm{a}$ \\
& $0.5 \%$ acetone & 8.8 & $7 \mathrm{~b}$ \\
II & Control b & 18.0 & 0 \\
& regular diet & 14.6 & $4 \mathrm{c}$ \\
& $0.5 \%$ acetone & 5.4 & $3 \mathrm{c}$ \\
SS & Control b & 14.6 & 0 \\
& regular diet & 26.0 & $29 \mathrm{~d}$ \\
& $0.5 \%$ acetone & 7.1 & $20 \mathrm{e}$ \\
& Control b & 26.0 & 0 \\
\hline
\end{tabular}

${ }^{\mathrm{a}} \mu$ moles $\mathrm{NADH} \times 10^{2} / \mathrm{min} / \mathrm{mgr}$ protein.

' In controls larvae were exposed to a $4 \%$ water solution for 40 minutes.

Larvae mortalities (transformed to angles) not followed by the same letter are significantly different at the 5\% level according to Tukey's test for multiple comparisons.

old B. oleae larvae reared on the $1 \%$ acetone diet showed a much higher mortality than did the regular and $0.5 \%$ acetone-fed larvae when they were transferred to the $0.025 \%$ pentenol diet lacking acetone. The viability of larvae fed on the artificial diet with $0.5 \%$ acetone for 7 days
Table 2 Percentage of adults recovered from larvae fed for a period of time (5, 6 or 7 days) on regular, on $0.5 \%$ acetone and on $1 \%$ acetone diets, and then transferred to a $0.025 \%$ pentenol diet. Larvae fed for 7 days on $0.5 \%$ and $1 \%$ acetone were additionally transferred to $0.5 \%$ acetone plus $0.025 \%$ pentenol or $1 \%$ acetone plus $0.025 \%$ pentenol, respectively

\begin{tabular}{lccc}
\hline Days of diet I & Diet I & Diet II & Adults recovery \% \\
\hline 5 & regular diet & $0.025 \%$ pentenol & 0 \\
& $0.5 \%$ acetone & & 0 \\
& $1 \%$ acetone & (regular diet) & 63 \\
6 & (regular diet) & 10 \\
& regular diet & $0.025 \%$ pentenol & 4 \\
& 0.5\% acetone & & 0 \\
& 1\% acetone & (regular diet) & 68 \\
7 & (regular diet) & 58 \\
& regular diet & $0.025 \%$ pentenol & $18 \mathrm{a} *$ \\
& 0.5\% acetone & & $10 \mathrm{~b}^{* *}$ \\
7 & $1 \%$ acetone & & 75 \\
7 & (regular diet) & (regular diet) & $65 \mathrm{c}^{*}$ \\
7 & $0.5 \%$ acetone & $0.5 \%$ acetone + & \\
& & $0.025 \%$ pentenol & $40 \mathrm{~d}^{* *}$ \\
& $1 \%$ acetone & $1 \%$ acetone + \\
\end{tabular}

${ }^{*} P<0.001,{ }^{* *} P<0.001$. Comparisons were made between treatments followed by the same number of asterisks.

Values shown are the means of four replicates, each of 100 larvae. Treatments that are not significantly different at the 5\% level (Tukey's test for multiple comparisons) are followed by at least one letter in common.

Table 3 Mean developmental times of egg-to-adult expressed in days together with their standard errors (given in parentheses). Individuals were kept on regular or acetone supplemented diet. Treatments not significantly different at the 5\% level (Tukey's test for multiple comparisons) are followed by at least one letter in common. The number of replicates was 5

\begin{tabular}{lccc}
\hline Genotype & Regular diet & $0.5 \%$ Acetone & $1 \%$ Acetone \\
\hline SS & $24.35(0.17) \mathrm{ab}$ & $24.56(0.098) \mathrm{ab}$ & $25.08(0.18) \mathrm{c}$ \\
FF & $24.35(0.064)$ ade & $24.7(0.077) \mathrm{acd}$ & $24.82(0.073) \mathrm{acd}$ \\
II & $24(0.057) \mathrm{e}$ & $24.5(0.045) \mathrm{d}$ & $24.56(0.068) \mathrm{d}$ \\
\hline
\end{tabular}


and subsequently transferred on diet with $0.025 \%$ pentenol, but without acetone, was $18 \%$, while larvae fed on a diet with $0.5 \%$ acetone for 7 days and then transferred on diet with $0.025 \%$ pentenol and $0.5 \%$ acetone exhibited $65 \%$ viability. When the acetone concentration was $1 \%$ instead of $0.5 \%$ and the experimental procedures were otherwise exactly the same, the viabilities were $10 \%$ and $40 \%$ respectively. This profound protection of $B$. oleae larvae against pentenol toxicity can be attributed to the presence of acetone, which continues to depress ADH activity from 7 th day to an adult eclosion. However, $\mathrm{ADH}$ activity can be restored when larvae are transferred to a diet without acetone and, as a result, significantly increased mortality provoked by pentenol appears. By this criterion, $B$. oleae larvae exposed to acetone are clearly deficient in ADH activity (Table 2). Both experiments confirm that, in $B$. oleae, acetone reduces larvae $\mathrm{ADH}$ activity in vivo, which in turn implies a parallel decrease in the mortality of these larvae when exposed to pentenol.

Having observed that $\mathrm{ADH}$ function is impaired by acetone, we examined the effect of acetone on homozygous ADH genotypes by determining the egg-to-adult viability and developmental time. In relation to adult recovery, the SS genotype fed on the regular diet showed significantly different higher adult recovery (Table 4) compared with that from the $1 \%$ acetone diet. Similar results were obtained with the II genotype. On the contrary, the FF genotype survived equally well on the control and on the acetone-supplemented diet. Almost the same conclusions can be drawn if homozygous $\mathrm{ADH}$ genotypes are compared with respect to developmental time and the effect of acetone treatment (Table 3). SS and II genotype raised on the control diet developed significantly faster compared with those on the $1 \%$ acetone diet while for the FF genotype, there was a tendency for a longer development time, although not significantly, for larvae fed with the $0.5 \%$ or the $1 \%$ acetone diets.

The effects of acetone on $\mathrm{ADH}$ allele frequencies (ADH-F, ADH-I, ADH-S) are summarized in Table 5. Statistical analysis (a chi-square test) indicated that ADH$\mathrm{S}$ and $\mathrm{ADH}-\mathrm{I}$ allele frequencies in individuals raised on a diet with $0.5 \%$ acetone varied significantly between the $\mathrm{P}$ and $\mathrm{F}_{2}$ generation $\left(\chi^{2}=5.346\right.$; d.f. $\left.=1 ; P<0.05\right)$, while the frequency of the ADH-F allele remained practically unaltered. Similar results were obtained in individuals on a diet that included $1 \%$ acetone $\left(\chi^{2}=8.503\right.$; d.f. $=1$; $P<0.05$ ). Frequencies of ADH alleles are not significantly different between $0.5 \%$ and $1 \%$ acetone treatments in each generation (G1 $\chi^{2}=2.468,0.20<P<0.30$. G2, $\left.\chi^{2}=0.644,0.70<P<0.80\right)$. Frequency of $F$ remains almost

Table 4 Mean percentage viability from egg-to-adult for the three $\mathrm{ADH}$ genotypes. Standard errors are given in parentheses. Individuals were kept on regular or acetone supplemented diet. Treatments not significantly different at the 5\% level (Tukey's test for multiple comparisons) are followed by at least one letter in common. The number of replicates was 8

\begin{tabular}{lccc}
\hline Genotype & Regular food & $0.5 \%$ Acetone & $1 \%$ Acetone \\
\hline SS & $0.57(0.038) \mathrm{a}$ & $0.52(0.007) \mathrm{a}$ & $0.43(0.029) \mathrm{bc}$ \\
II & $0.46(0.022) \mathrm{bd}$ & $0.42(0.038) \mathrm{be}$ & $0.38(0.025) \mathrm{e}$ \\
FF & $0.36(0.024) \mathrm{e}$ & $0.34(0.03) \mathrm{e}$ & $0.37(0.027) \mathrm{e}$
\end{tabular}

Table 5 Effect of acetone on $\mathrm{ADH}$ allele frequencies (ADH-F, tained on regular food $(\mathrm{P})$ and for two consecutive generations ( $\mathrm{F}_{1}$ and $\mathrm{F}_{2}$ ) on food with $0.5 \%$ or $1 \%$ acetone

\begin{tabular}{|c|c|c|c|c|c|c|}
\hline$G$ & & No. & $A D H-F$ & $A D H-I$ & $A D H-S$ & \\
\hline $\mathrm{P}$ & Regular diet & 228 & 0.465 & 0.309 & 0.226 & $\begin{array}{c}\mathrm{P}-\mathrm{F}_{2} \\
\text { alleles I and S }\end{array}$ \\
\hline $\mathrm{F}_{1}$ & $0.5 \%$ acetone & 206 & 0.451 & 0.297 & 0.252 & $\begin{array}{c}\chi^{2}: 5.346 \\
\text { d.f. : } 1\end{array}$ \\
\hline $\mathrm{F}_{2}$ & $0.5 \%$ acetone & 200 & 0.485 & 0.243 & 0.272 & $P<0.05$ \\
\hline $\mathrm{P}$ & Regular diet & 228 & 0.465 & 0.309 & 0.226 & $\begin{array}{c}\mathrm{P}-\mathrm{F}_{2} \\
\text { alleles } \mathrm{I} \text { and } \mathrm{S}\end{array}$ \\
\hline $\mathrm{F}_{1}$ & $1 \%$ acetone & 206 & 0.488 & 0.245 & 0.267 & $\begin{array}{c}\chi^{2}: 8.503 \\
\text { d.f. }: 1\end{array}$ \\
\hline $\mathrm{F}_{2}$ & $1 \%$ acetone & 239 & 0.462 & 0.241 & 0.297 & $P<0.01$ \\
\hline
\end{tabular}

$\mathrm{G}=$ generations, No. = sample size .

the same between G0 and G2 for both acetone concentrations $\left(0.5 \%, \chi^{2}=0.3000 .30<P<0.50 .1 \%, \chi^{2}=0.460\right.$, $0.30<P<0.50)$.

Although there does not seem to be too good a relationship between the changes in viability and egg-toadult developmental time in the two experimental conditions and the observed changes in gene frequencies when individuals have been exposed to these conditions for two generations, we must keep in mind that $\mathrm{ADH}$ polymorphism is governed by a frequency dependent mechanism which alters egg-to-adult viability and developmental time when all genotypes are growing in the same cup of food (Cosmidis et al, 1999).

\section{Discussion}

The ADH polymorphism in B. oleae (formerly Dacus oleae) was first detected by Bush and Kitto (1979) in a preliminary survey of allozyme variation in this most important pest of the Mediterranean basin. Bush and Kitto found two alleles, fast and slow (F and S), at approximately $40 \%$ and $60 \%$ frequencies in a natural population in Greece. Subsequently, Zouros et al (1982) found a third allele (Intermediate, I) in a laboratory colony maintained on artificial larval food. Further examination showed that in colonies of the olive fruit fly $B$. oleae, originating from several independent populations and maintained on an artificial substrate, the ADH-I allele of the ADH locus, whose frequency in natural populations is less than $1 \%$, rises in frequency to about $30 \%$ within four generations of artificial rearing, that of ADH-S, which is most common in natural populations, suffers a compensatory decrease from $60 \%$ to $30 \%$ whereas that of $\mathrm{ADH}-\mathrm{F}$ is only slightly affected. Since then, a considerable amount of information about the ADH locus has been obtained (Cosmides et al, 1997; Cosmidis et al, 1999; Mazi et al, 1998b; Benos et al, 2000; Goulielmos et al, 2001). The B. oleae ADH selection pressure appears to be amongst the largest ever reported for allozymes, and is only comparable to selection pressure suffered by plants growing on soils contaminated by heavy metals (Jain and Bradshaw, 1966).

In flies of $B$. oleae homozygous for a given electrophoretic allozyme, three forms of $\mathrm{ADH}$ activity termed 'isozymes' are observed after electrophoresis. We attempted 
to explain this pattern of electophoretic variation in the light of a model advanced for the origin of the multiple forms of $\mathrm{ADH}$ in D. melanogaster (Schwartz et al, 1975; Schwartz and Sofer, 1976).

This model makes a very specific prediction. If we rear B. oleae larvae on food supplemented with acetone, the relative proportions of the isozymes should change. Specifically, the less cathodal forms of the enzyme should increase at the expense of the most cathodal one. As shown in Figure 2, this hypothesis was fully confirmed. The exposure of $B$. oleae larvae to acetone induces an 'interconversion' of $\mathrm{ADH}$ isozymes towards the more electronegative forms. Two other predictions of the model have been confirmed. First, since homozygous genotypes for the three known alleles each display three isozymes zones after electrophoresis, it is expected that each would show a similar response to being fed on diet with acetone. Indeed, we found that in genotypes homozygous for each of the ADH variants, there was a change in the proportions of the three isozymes, such that the less cathodally migrating forms seemed to increase in activity, whereas the most cathodally migrating form lost activity after addition of acetone to the food. Second, a decrease in the activity of $\mathrm{ADH}$ is expected as a function of acetone treatment. The elevated ability of larvae fed with acetone diet to withstand pentenol reflects a decline in $\mathrm{ADH}$ activity, induced by acetone, and consequently strongly suggests that acetone provokes in vivo a considerable restriction of ADH activity. Larval growth was restricted in an acetone concentration of $1 \%$ for the SS and II genotypes while the FF genotype remained unaffected. A prolonged developmental time for all homozygous genotypes was recorded for both acetone concentrations used and was statistically significant for genotypes II (0.5 and 1\%) and SS ( $1 \%)$.

The selection pressure exercised on the ADH polymorphism by acetone was in the opposite direction from that always observed on a regular diet. The ADH-S frequency increased and that of ADH-I decreased. The F allele remained unchanged. A limitation in this experiment might be that we maintained the B. oleae laboratory populations exposed to acetone only for two generations. However, a particularly strong piece of evidence, already available, leads us to believe in the direct action of selection at the $\mathrm{ADH}$ locus of $B$. oleae during laboratory rearing. In the original population used in this experiment, we have not observed any substantial change of $\mathrm{ADH}$ allele frequency over a period of 50 generations. We feel that it is rather unrealistic to consider that after 50 generations of $\mathrm{ADH}$ equilibrium, the presence of acetone in the food of larvae and, simultaneously, a significant change in ADH S and I alleles' frequencies can be attributed to random genetic drift or to mutation, since we have already shown that there is a correlation between acetone and ADH activity, egg-to-adult viability and developmental time, parameters of an insect life history which are selectively very important in their expected effects on the ADH polymorphism.

The response of the $\mathrm{ADH}$ polymorphism to acetone treatment provides valuable clues for further search for a rearing protocol and qualitative performance standards in $B$. oleae laboratory populations. Modification in the dietary regime, via acetone utilization, may improve the quality of artificially reared populations in such a way that may soon become more suitable for the purposes of biological control (SIT) of B. oleae.

\section{Acknowledgements}

The authors would like to thank Mrs D Loukas for typing the manuscript, Dr A Katsiotis for assistance with the Photoshop software, and two anonymous reviewers for helpful suggestions.

\section{References}

Benos P, Tavernarakis N, Brogna S, Thireos G, Savakis C (2000). Acquisition of a potential marker for insect transformation: isolation of a novel alcohol dehydrogenase gene from Bactrocera oleae by functional complementation in yeast. Mol Gen Genet 263: 90-95.

Bradford M (1976) . A rapid and sensitive method for the quantification of microgram quantities of protein utilizing the principle of protein -dye binding. Anal Biochem72: 248.

Bush GL, Kitto GB (1979). Research on the genetic structure of wild and laboratory strains of olive fly. F.A.O. Report, Development of Pest Management Systems for Olive Culture Program. F.A.O. of the United Nations, Rome.

Cosmides N, Loukas M, Zouros E (1997). Differences in fitness components among alcohol dehydrogenase genotypes of the olive fruit fly (Diptera: Tephritidae) under artificial rearing. Ann Entomol Soc Am 90: 363-371.

Cosmidis N, Loukas M, Zouros E (1999). Rarer need not be better if commoner is worse: frequency-dependent selection for developmental time at the alcohol dehydrogenase locus of the olive fruit fly, Bactrocera oleae. Evolution 53: 518-526.

Economopoulos AP, Avtzis N, Zervas G, Tsitsipis J, Haniotakis G, Tsiropoulos G et al (1977). Experiments on the control of the olive fly, Dacus oleae (Gmel) by the combined effect of insecticides and releases of gemma-ray sterilized insects. $Z$ ang Ent 83: 201-215.

Economopoulos AP, Loukas M (1986). ADH allele frequency changes in olive fruit flies shift from olives to artificial larval food and vice versa, effect of temperature. Entomol Exp Appl 40: $215-221$

Everse J, Zoll EC, Kahan L, Kaplan NO (1971). Addition products of diphosphopyridine nucleotides with substitutes of pyridine nucleotide-linked dehydrogenases. Bioorg Chem 1: 207.

Garrido JJ, Dorado G, Barbancho M (1988). Participation of Drosophila melanogaster alcohol dehydrogenase (ADH) in the detoxification of 1-pentene-3-ol and 1-pentene-3-one. Heredity 61: 85-91.

Goulielmos GN, Cosmidis N, Loukas M, Tsakas S, Zouros E (2001). Characterization of two alcohol dehydrogenase (Adh) loci from the olive fruit fly, Bactro cera (Dacus) oleae and implications for Adh duplication in Dipteran insects. J Mol Evol 52: 29-39.

Jain SK, Bradshaw AD (1966). Evolutionary divergence among adjacent plant populations. I. The evidence and its theoretical analysis. Heredity, 21: 407-441.

Mazi VE, Cosmidis N, Clonis YD, Loukas M (1998a). Purification of alcocol dehydrogenase from four genotypes of the olive fruit fly Bactrocera (Dacus) oleae. Biotech Prog 36: 259-269.

Mazi V, Cosmidis N, Loukas M, Clonis Y, Zouros E (1998b). Biochemical differences between products of the ADH locus in olive fruit fly Bactrocera oleae. Biochem Genet36: 259-269.

Papel I, Hederson M, Herewege J, Van David J, Sofer W (1979). Drosophila alcohol dehydrogenase activity in vitro and in vivo: effects of acetone feeding. Bio Chem Genet 17: 553-563.

Poulik M D (1957). Starch gel electrophoresis in discontinuous systems. Nature, 180: 1477.

Schwartz M, Gerace L, O Donnell J, Sofer W (1975). Droshophila alcohol dehydrogenase: origin of the multiple forms. In: Third 
International Conference on Isozymes, Vol I, Academic Press: New York.

Schwartz M, Sofer W (1976). Dietary induced alterations in the distribution of the multiple form of alcohol dehydrogenase in Drosophila. Nature 263: 129.

Sofer W, Hatkoff MA (1972). Chemical selection of alcohol dehydrogenase negative mutants in Drosophila. Genetics 72: 545 .
Tsitsipis JA (1983). Optimization of the hologidic diet for the larvae of the olive fruit fly. In Cavalloro R (ed) Fruit Flies of Economic Importance. Proceedings of the CEC/IOBC International Symposium, 1982, Athens, Greece. Balkema, Rotterdam, The Netherlands. pp 423-428.

Zouros E, Loukas M, Economopoulos AP, Mazomenos B (1982). Selection at the alcohol dehydrogenase locus of the olive fruit fly Dacus oleae under artificial rearing. Heredity 48: 169-185. 